\title{
预后控制外科: 从理论到实践
}

\author{
刘荣”, 刘渠, 王斐, 王子政, 张涛, 张恭 \\ 解放军总医院肝胆外二科, 全军肝胆外科研究所, 北京 100853 \\ *联系人, E-mail: liurong301@126.com \\ 2019-02-22 收稿, 2019-03-11 修回, 2019-03-12 接受, 2019-03-27 网络版发表 \\ 军事医学创新专项(13CXZ029)资助
}

\begin{abstract}
摘要当前以互联网、大数据和人工智能为代表的信息技术的应用将外科实践提升到了智能化的新高度, 以干预者经验为主导的传统医学和过度倚重干预手段的循证医学受到了新的冲击和挑战. 为了更好地适应 医学科技的飞速发展和医疗需求的不断提高，根据长期的肝胆胰外科临床实践，本文提出了预后控制外科 理论。该理论以患者最优预后为根本目标，通过择优千预者、千预手段、千预时机的最佳组合来预控疾病 风险. 随着人工智能技术的逐渐兴起，本文提出了智能外科的概念，预控外科在其基础上，提倡应用医学影 像识别与预测、三维重建与手术规划、术中导航和机器人手术系统为代表的人工智能等信息技术方法, 促 进外科诊疗的快速、均衡发展。在预控外科理论和方法的指导下，本研究团队创立了微创解剖性肝切除技 术、个体化胰腺手术入路体系、模式化肝切除方法、单针全层胇肠吻合技术和胰腺端端吻合技术，提倡胰 腺癌整块切除, 并归纳总结出了外科四大核心策略: 控血、入路、切除、重建, 以最小损伤实现患者的最大 获益.
\end{abstract}

关键词预控外科, 控血技术, 入路选择, 切除技术, 重建技术, 人工智能

随着互联网、大数据时代的到来，以及人工智能 等信息化技术在医疗领域的逐渐应用, 以干预者经验 为主导的传统医学和过度倚重干预手段的循证医学受 到了新的冲击和挑战 ${ }^{[1]}$. 随着医学科技的飞速发展, 新 的疾病诊疗方法层出不穷，这些高科技的技术手段给 医学带来的不仅仅是先进的诊疗技术和设备, 更多的 是对整个诊疗理念的更新和挑战. 因此, 有必要将干预 者的临床经验与高等级循证医学证据和人工智能等信 息技术相融合，通过有效地控制疾病风险使患者获得 最佳的治疗效果. 本研究团队在长期的肝胆胰外科临 床实践中提出了预控外科理论, 通过建立微创肝胆胰 手术和智能化肝胆胰外科诊疗等方法, 实现了“微创手 术常规化”和“复杂手术简单化”，力求以最小的医疗创 伤实现患者最大的临床获益.

\section{1 预后控制外科理论}

\section{1 概念}

根据当前的科技发展形势及医学发展方向，本文 提出了预后控制外科的理念，即利用神经网络、深度 学习、大数据以及图像学等信息技术，预测外科疾病 风险，择优干预者、干预手段、干预时机及其最佳组 合, 并加以控制, 实现预后全程最优, 使患者得到最好 的治疗效果 ${ }^{[2]}$, 简称为预控外科. 预控外科是将最新医 学科技与理论赋能外科实践的产物, 形成了外科疾病 风险管理的基本理论框架，能够为疾病临床决策制 定、临床干预实施和预后模型分析提供了关键性思路. 干预者只有将预控外科的理念贯穿于围手术期的各个 方面，才能通过对诊疗过程的流程再造实现预后全局 
最优的最终目的.

预控外科不仅是一种创新的医疗理念, 也有一套 完整的理论体系和实施方法, 能够从疾病风险管理的 各个阶段、从临床干预的各个方面实现风险预控及患 者获益最大化. 预控外科理念指导下的临床决策不同 于包括循证医学和多学科团队协作(multiple disciplinary team, MDT)在内的当代外科决策, 而是一个多种干 预信息相互协同的高维度决策体系(图1). 高维干预信 息协同模型用高维网状决策代替了传统的二维树状决 策, 有利于实现干预全局、预后全程的优化; 通过对干 预者能力的分型突破了按学科分类的局限性，有利于 发挥诊疗主体的能动性; 通过将风险干预前置, 有利于 用目标明确的预后控制代替既往诊疗模式中单纯结局 预测. 预控外科也在不断发展完善, 能够紧跟医学科技 的飞速发展, 根据患者日益增长的治疗需求, 充分利用 人工智能等信息化成果来服务患者.

\section{2 预控外科四要素}

根据外科诊疗的临床特点和预控外科的实践过程, 我们将疾病风险、干预者、干预手段和干预时机称为 预控外科的四要素. 只有在临床中把握住这 4 个关键点 才能有的放矢地提高外科诊疗水平, 才能使患者在医 学的发展进步中充分获益, 才能实现患者的最佳预后. (1) 疾病风险既包括了目标疾病本身的可能造成的不 良后果，也包括患者自身基础疾病可能带来的风险. 以
肝癌肝硬化为例, 严重的肝硬化程度会显著增加肝癌 切除术中出血和术后肝功能障碍的风险. 外科治疗的 目的就是以最小的干预风险来实现最大程度的干预对 象的控制. (2) 干预者是诊疗过程的主体，干预者的经 验和能力决定了干预时机和干预手段的选择和实施. 作为预控外科的主导者与执行者, 干预者不仅需要从 大量的临床实践中提高临床决策和诊疗的能力，还需 要在医疗科技、智能医学的发展中与时俱进、不断创 新，善于利用大数据和人工智能等信息技术手段来提 高干预的有效性和时效性 ${ }^{[3]}$. 由于干预者是人，医疗的 对象也是人，干预者还需要善于控制自身情绪、通过 人文关怀来满足患者的心理需求、提高诊疗效果. (3) 干预手段是完成疾病诊疗的直接工具，干预手段的合 理选择和恰当使用是实现患者良好预后的必要条件. 当前以人工智能为代表的信息技术已经打开了智能医 学革命的大门, 给外科领域带来重大的变革, 不仅实现 了对现有诊疗方法的更新换代，还带来了更多创新性 的诊疗手段 ${ }^{[4]}$, 通过深度的人机交互, 最大限度地发挥 干预者与干预手段的效能，实现可靠、高效以及个体 化的精准治疗 ${ }^{[5]}$. (4) 干预时机是干预者利用干预手段 进行疾病风险管理的时间节点. 干预时机是“看不见、 摸不着”的主观因素，恰当的干预时机有助于干预者和 干预手段发挥出最佳的干预效能，在临床工作中干预 者需要实时判断疾病风险、干预风险与患者获益之间 的关系，通过评估患者获益与风险之间的最大化差值

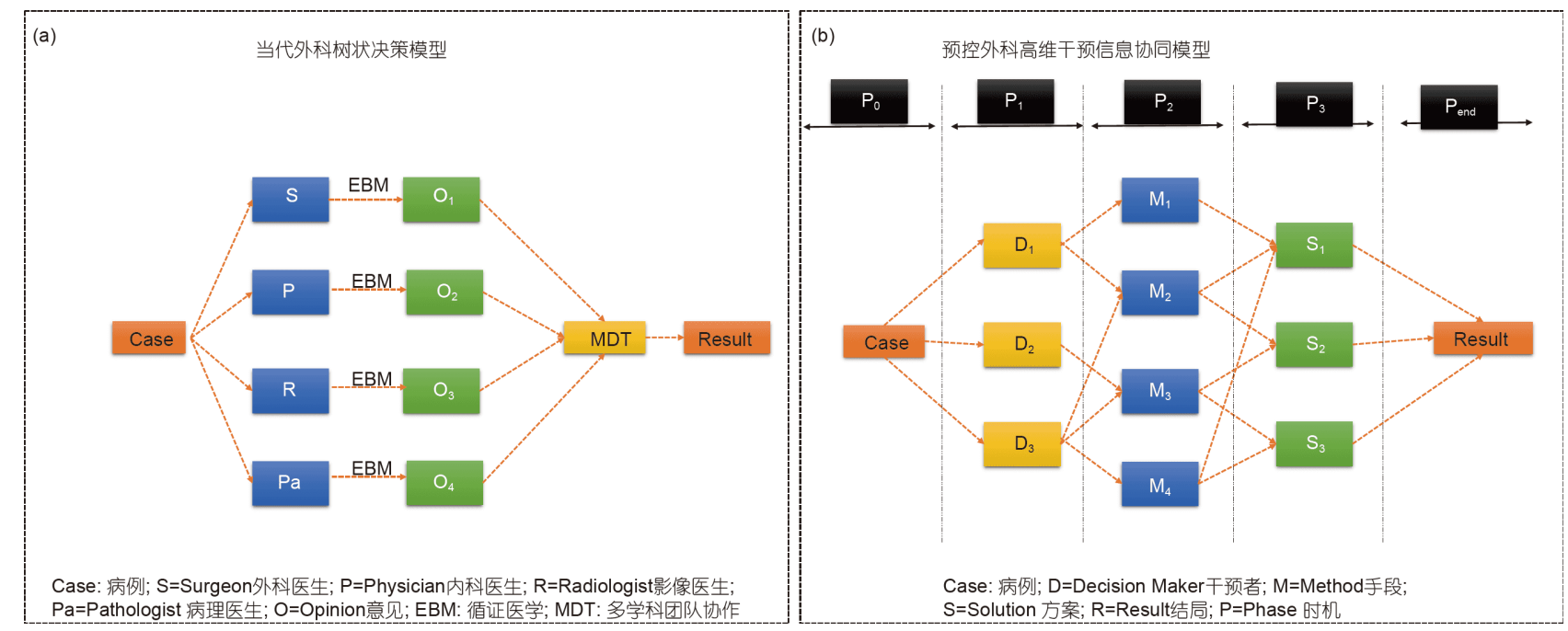

图 1 (网络版彩色)预控外科的临床决策. (a) 当代外科树状决策模型; (b) 预控外科高维干预信息协同模型

Figure 1 (Color online) Clinical decision making in prognostic control surgery. (a) Decision tree model in contemporary surgery; (b) high dimensional collaborative model in prognostic control surgery 
来决定最合理的干预时机.

\section{3 患者预后是最终目标}

预后的英文是Prognosis，其中Pro-是预先，-gnos是 知道, 因此组合在一起就是预先知道, 在医学上也就是 预见疾病发展情况的意思. 预后并非愈后, 而是指预测 疾病的可能病程和结局, 既包括了疾病的发展结果, 也 具有时间上的延展性. 2000多年前希波克拉底在《论 预后》中认为要通过临床观察和分析来判断疾病预后, 并且预后情况是衡量医术水平高低的一种尺度 ${ }^{[6]}$.

科技进步是推动医学发展的强大动力, 然而目前 对医学的研究似乎又过度聚焦于技术本身，过于强调 新技术、新业务的应用，而轻视了医疗的根本目的在 于提高疾病治疗的质量, 改善患者的预后. 患者预后是 评判干预质量最重要的标准, 最优的患者预后是疾病 诊疗中始终应该坚持的目标. 随着医学亚专业越分越 细、干预手段选择越来越多样，不同干预者所精通的 专业领域和干预手段也越来越细化，以患者预后为根 本导向，选择最优的干预者、干预手段、干预时机及 其组合是优秀的临床干预者所必备的素质.

\section{4 疾病风险管理是实现过程}

从预控外科的理论来看医疗行为的过程就是寻求 疾病风险最小化的过程, 这个过程就叫做疾病风险管 理. 风险管理过程分为两个部分: 风险评估与风险控 制. 在风险评估过程中, 首先需要进行全面的风险识 别，包括疾病风险、干预者、干预手段及干预时机相 关的风险. 在进行全面的风险识别后, 要对所识别的风 险进行评价. 在风险评价的过程中, 应当注意疾病风 险、干预者风险、干预手段风险、干预时机风险并不 是独立存在的, 而是相互联系、相互作用、相互转化 的. 风险控制的过程是预控外科的核心操作过程，目的 在于通过预防和控制措施，减少风险引起的不良后果 的严重程度 ${ }^{[7]}$. 既往的治疗理念将重点放在了疾病风 险上，预控外科理论则首次将干预者、干预手段及干 预时机风险的概念提出, 强调疾病风险和干预风险是 相通的，通过全面、切实的风险管理将疾病风险和干 预风险控制在最小范围内, 是预控外科的实现过程, 也 是患者获得最优结局的保证.

\section{5 干预三因素的优化组合是实现手段}

在临床实践中，医疗干预需由干预者通过干预手
段在恰当的干预时机发挥作用, 干预者、干预手段、 干预时机是影响患者预后的三个最重要的因素. 干预 三因素之间不是孤立存在的，而是相互联系、相互作 用、相互影响，需要对干预者、干预手段和干预时机 的不断优化和配置组合才能实现患者的最优预后和最 佳获益.

由于教育背景和工作平台的差异, 不同干预者的能 力和经验之间存在着很大的差异, 对于干预手段的掌握 和干预时机的把握各不相同. 现有的医学模式过度强调 了最佳干预手段的选择,而忽视了干预者能否熟练掌握 该项技术方法, 缺乏对干预者自身专业技能和经验的评 估，容易进人干预者和干预手段无法匹配的误区. 预控 外科首次将干预者的能力和状态纳人评价体系, 通过第 三人称视角, 以干预者对不同干预手段的掌握情况和情 绪状态为依据, 更加客观地对干预手段和干预时机进行 有效选择. 此外, 以机器学习、图像识别、术中导航为 代表的人工智能等信息化技术的飞速发展, 不仅促进了 干预手段的不断优化，也对干预者提出了更高的要求. 干预者需要不断与时俱进, 调整诊疗理念, 将新型的干 预手段应用到干预过程中，并制定与之相应的干预时 机, 最终实现干预三因素的优化组合.

\section{2 预控外科的方法}

人工智能(artificial intelligence, AI)正在逐渐兴起 成为颠覆各个领域的强大动力，用AI技术解决行业问 题最重要的是应用场景, 而AI 在医疗服务的核心应用 场景是解决优质医疗资源不足的问题. 如何通过AI在 医疗机构的应用, 提升大医院的生产效率, 增强基层医 疗的服务水平，在疾病的诊断、预测、治疗和管理服 务上发挥AI的优势, 是我们当前面临的主要问题. 根据 外科学及人工智能的趋势发展, 本研究团队提出了使 用人工智能辅助或替代人类进行外科诊断、治疗的智 能外科的概念 ${ }^{[5]}$. 预控外科在智能外科的基础上, 强调 利用人工智能等信息化方法，打破传统的干预者、干 预手段和外科患者的三方博弯关系，通过其纽带作用， 将原有的三者结合为一个新的整体，提升了彼此的交 互效率，通过新的生产关系创造出新的生产力(图2), 有助于改善外科治疗的不确定性和外科水平发展的不 均衡性.

\section{1 智能医学影像识别与预测}

智能医学影像识别旨在通过深度神经网络及卷积 


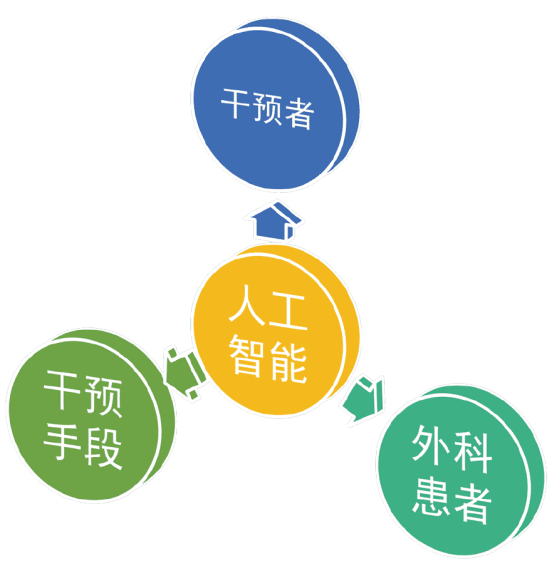

图 2 (网络版彩色)预控外科下的新型外科关系

Figure 2 (Color online) New surgical relationships under prognostic control surgery

学习技术, 对CT (computed tomography)、MR (magnetic resonance)、手术视频等常用的医学影像进行分 析处理, 其目的是提高影像识别的效率和质量, 进而为 诊断和治疗提供帮助.

目前智能医学影像的识别主要集中在器官识别、 血管分割、病理切片分析、基于医学影像的病理分 析、手术视频内容解析等几个方面. 其中将目标区域 从图像中识别出来是智能医学影像处理的首要和核心 步骤. 为了实现准确的目标识别出现了多种人工智能 方法, 例如通过人工标记训练机器再进行机器自主学 习的方法, 或者通过与临近大器官的空间关系来进行 图像识别, 或使用迭代方法通过两个神经网络先后进 行粗略分割及精细分割来识别目标区域. 这些方法各 有优劣, 简单的方法往往识别效率不高, 而较为复杂的 方法具有运算量过大, 对硬件要求高的缺点(表1).

手术视频的解析是智能外科新发展起来的另一个 分支, 通过手术视频的解析, 可以使机器理解当前的手 术操作, 从而对手术操作的细节进行提示并且帮助术 者进行下一步操作的合理选择. 视频解析是一种通过
自学习功能，不断搜集与理解环境信息和自身的信息， 在实践中不断地充实知识库, 分析判断和规划自身行 为的制造系统. 在未来, 通过手术视频解析技术结合 3D打印技术等, 可以协助加速外科医学生的手术学习, 缩短外科医生的培养周期.

由于医学数据获取途径和获取条件单一, 训练数 据缺乏且较难获取, 目前智能医学影像识别仍然存在 疾病谱覆盖窄、诊断准确率仍需提高等诸多问题. 如 何整合资源, 解决医学数据获取困难的问题, 满足人工 智能所需要的大数据量, 是智能医学影像研究中面临 的紧要问题. 目前本团队利用 $v g g 16$ 定位联合inception 识别的两步法卷积神经网络, 优化图像后处理方法, 并 通过 $Z$ 轴连续性篮选进一步去除假阳性结果, 显著提高 了胰腺囊腺瘤分型诊断的准确率，有利于手术决策的 选择 ${ }^{[8]}$.

\section{2 三维重建与手术规划}

自20世纪90年代美国首先提出“可视人计划”(Visible Human Project) ${ }^{[9]}$ 以来, 三维虚拟影像技术迅速发展, 其在外科领域的应用带来了一场新的外科学革命. 三 维虚拟影像技术在高精度 $\mathrm{CT}$, MR数据的基础上, 通过 数字化三维重建, 形成立体、完整的器官组织图像, 能 够真实地再现器官组织的精细解剖结构, 可以帮助医 生更好的理解、分析病变情况及制定手术方案，从而 使手术可以更加安全有效的进行, 降低术中风险, 改善 患者预后.

根据病变部位的平面数据进行三维重建, 能够真 实反映病变位置以及与血管、脏器的解剖关系. 通过 对立体影像资料中的病灶进行精确观察判断, 为医生 提供手术方案的决策支持，在手术规划系统的帮助下， 使术前评估更为精准，手术方案更加科学、个体化. 同 时帮助医生与患者之间能进行更加直观的沟通, 并且 对辅助术后观察及效果评估有重大意义.

表 1 三种智能医学影像识别方法的区别

Table 1 The difference of three intelligent medical image recognition methods

\begin{tabular}{clll}
\hline 项目 & \multicolumn{1}{c}{ 机器学习模型训练法 } & \multicolumn{1}{c}{ VOI(Volume Of Interest)法 } & 迭代法 \\
\hline 原理 & 将经过人工标注后的图像训练机器学习模型, & 先对小器官临近的大器官进行定位, 然后根据 & 使用迭代方法, 通过两个神经网络 \\
& 获得器官的形状及位置后, 再在位置附近对 & 二者的关系确定包含目标器官的容积, 进而 & 先后进行粗略分割及精细分割 \\
& 器官进行分割 & 再次进行分割操作 & \\
优点 & 简化了算法流程 & 对小器官识别效果好 & 对不同器官均有较好的识别率 \\
缺点 & 对小器官的识别率不高 & 识别效率低 & 运算量过大, 对硬件要求高 \\
\hline
\end{tabular}


在未来发展中，三维重建技术将会加人更多的信 息, 如运动补偿、深度信息、压缩感知等, 以更加符合 实际情况. 其还可以与MR (mix reality)技术及术中导航 技术相结合，将术中手术部位的三维重建图像实时投 影在手术区域, 使其随着术者的手术操作, 患者的实时 状态改变而随时调整，使得术者更加直观地了解手术 部位的解剖结构及其实时改变，从而使得手术过程更 加简单直接, 降低手术难度.

\section{3 术中导航}

术中导航技术是一种由智能机器和人类专家共同 组成的人机一体化智能系统，其利用计算机、声学、 放射、光学和电磁等技术, 在医生手术过程中, 通过计 算机屏幕提供直观的、图像融合的关键组织或脉管位 置等信息在内的手术场景的图像, 以辅助手术实施的 一种计算机辅助方法 ${ }^{[10]}$. 术中导航是一门新兴的医学 与工学交叉的技术, 是人机一体化的混合智能系统, 将 扩大、延伸和部分地取代人类专家在制造过程中的脑 力劳动. 通过术中导航, 可以降低手术难度、提高手术 精度、减少手术并发症, 可以使复杂手术简单化, 便于 一些高难度手术的开展和推广. 手术导航系统最早应 用于神经外科领域、后来随着导航技术的不断发展, 其临床范围已逐步扩展到脊柱外科、耳鼻喉科、整形 外科等 ${ }^{[11]}$, 不仅提高了手术质量, 并使得之前无法完成 或者风险极大的手术变得可行.

术中导航技术包括以下几项关键技术: 手术计划 系统、空间定位技术及图像配准. 计划系统可以在手 术前在虚拟空间里进行三维手术模拟, 制定出较为完 备的手术计划, 并且可以在手术过程中实时对比手术 模拟. 空间定位技术可以建立病人、手术场景和术前 影像三者之间紧密的相互关系. 动态图像配准是术中 导航最重要的一步, 在手术过程中术者及手术目标区 域的组织器官处于相对位置不断变动的过程, 经过动 态配准使机器不断追踪识别变换位置的目标.

在手术过程中，为了实现与术前图像融合与精确 配准, 需要选择使用不同的术中导航系统. 导航系统在 使用过程中, 精度要求至少要高于 $1.5 \mathrm{~mm}^{[12]}$. 目前的导 航系统分为三类: 光学导航系统、电磁导航系统及混 合导航系统. 光学导航系统跟踪的手段主要是通过物 体对红外线的反射, 具有跟踪精度较高、作用距离较 远等优势, 但是受到其他物体遮挡则无法正常工作. 电 磁导航系统没有光学系统“遮挡”的限制, 在人体内也
可以追踪, 但是作用距离有限. 混合导航系统充分利用 了以上两者的优点, 尽可能地克服了两者缺点, 但是因 为设备较多, 增加了系统的复杂度, 容易产生系统误差, 且价格昂贵 ${ }^{[11]}$. 在未来发展中, 术中导航将从如何降低 延迟率、提高准确度、提高建模效率等几个核心技术 方面不断研究, 解决术中导航技术发展与临床实际应 用需求不匹配的现状，发展出具有我国自主知识产权 的术中导航设备.

\section{4 远程、智能化机器人手术系统}

机器人手术系统起源于远程手术，2000年美国Intuitive Surgical公司成功开发了具有立体可视化和人机 交互界面的Da Vinci外科手术系统 ${ }^{[13]}$, 开启了机器人在 外科领域的尝试，为外科手术的远程化、智能化的操 作提供了一个平台. 当前计算机、远程通讯、互联网 及手术机器人技术的发展为远程手术的实现奠定了基 础. 远程机器人手术一方面可使患者尽早得到最优外 科医生的治疗, 有利于把握最佳的治疗时机, 优化医疗 资源配置; 另一方面, 手术医生可以连续为多名位于不 同地方的患者进行远程手术, 有助于提升救治效率. 但 是远程手术技术仍然处在动物实验与临床试验阶段, 仍然面临着信息传输延迟、机器人手术助手与器械护 土培养成本高、机器人手术方式尚未统一、适应症受 限等一系列问题.

智能化是机器人手术系统发展的核心和难点, 手 术机器人不仅仅是精细手术的实施工具, 而应该通过 分析大量、高维度的实时数据, 利用更多更先进的传 感器, 主动识别病灶位置、制定手术方案, 在手术医生 的监督下自动实施手术, 实现手术“全机器人化”. 从而 解放外科医生, 使其从外科手术的实施者转变为一个 监督者.

本团队自开展机器人手术以来，在大量手术经验 积累的过程中实现了术式和理论的不断创新: (1) 截至 2019年1月20日，本团队完成了单中心 3000 例机器人肝 胆胰外科手术, 占到全国机器人肝胆胰手术总量的 $32 \%$, 拓宽了机器人手术范围和适应症, 不断突破机器 人肝胆胰手术的禁区. (2) 实现术式和理论创新, 提出 了便于钩突显露和胃肠吻合的肠系膜血管左、右开孔 法——LR孔”法 ${ }^{[14,15]}$; 完成了世界首例机器人后腹腔 镜胰腺手术 ${ }^{[16]}$; 利用创新性桥梁合拢理论提出了“主胰 管架桥修复”和“荣氏胰腺中段切除术”, [17 19]等创新术 式. (3) 针对机器人手术系统人在回路的特征，以专家 
经验赋能新一代手术机器人设计, 针对当前机器人手 术系统力反馈缺乏、智能化程度较低等不足, 以丰富 临床经验提出研发需求并进行验证, 参与建设新型智 能化机器人手术平台. (4) 完成全球首例基于 $5 G$ 网络的 远程机器人手术, 利用 $5 \mathrm{G}$ 传输技术和国产机器人手术 系统, 对实验动物实施远程肝切除手术, 验证了远程手 术在 $5 \mathrm{G}$ 技术条件下的可行性.

\section{3 预控外科的核心策略}

\section{1 控血技术}

控制出血是外科的永恒主题和核心技术环节，实 施有效的血流控制是决定手术成败以及患者预后的关 键因素. 特别对于微创手术来说, 在放大的镜头下一旦 出现较大的出血不仅使正常的解剖层次变得难以分辨, 增加了手术的难度, 更是对术者心理的极大考验, 是导 致手术中转开腹和术后严重并发症的主要因素, 因此 微创手术更强调对术中出血的控制. 早期由于对微创 外科技术特点缺乏深刻的认识，开腹手术时能够自如 运用的控血方法在微创条件下难以应用, 以肝脏切除 为代表的复杂手术微创化进程举步维艰. 我们通过总 结腹腔镜和机器人肝切除手术经验，根据肝脏血管分 布规律及微创手术特点, 提出了肝段供血的流域学说 理论，以“防止出血”的肝脏血流预控理论代替了“术中 止血”的传统理念，建立了预先显露和控制拟切除区域 血管的肝切除控血技术 ${ }^{[7,20,21]}$.

肝脏血流预控理论不拘泥于某一种具体的阻断方 法，而是强调综合运用控血技术对拟切除肝脏区域血 流实施预先控制. 首先, 结合术前影像学资料或三维重 建模型, 判断病灶部位与周围血管分布情况, 预先制定 肝脏拟切除区域及相应的控血方案. 其次, 在处理人肝 血流时，应根据拟切除肝脏区域的范围游离出供血的 肝蒂予以预先阻断，一方面可以提前控制切肝过程中 肝脏切面的出血，另一方面也可以防止肿瘤的医源性 播散. 在实践中, 由于肝动脉及门静脉的解剖变异并不 少见, 我们建议利用微创手术中放大视野以及精细化 操作的优势行肝门部管道鞘内解剖, 从而可以避免因 解剖变异而引起的血管和胆管的损伤. 在肝硬化或肿 瘤压迫而导致的肝门解剖困难的情况下可以改行鞘外 解剖或行直线切割闭合器“集束式”离断，以避免医源 性的肝门部管道损伤 ${ }^{[22]}$.

我们在大量的肝切除手术中发现传统的Couinaud
分段有其局限性，经常会出现区域肝蒂阻断后缺血线 与Couinaud分段不符的现象，周围肝蒂共同给肝段供 血的流域学说理论更加符合肝脏供血的解剖性特 点 ${ }^{[21]}$. 因此在肝切除过程中我们建议行辅助性第一肝 门阻断，这样可以阻断邻近肝蒂交通支对拟切除肝脏 的供血, 进一步提高术中控血效果. 由于微创镜头下视 角和操作受限, 对于出肝血流的阻断, 术者需要根据手 术需要及自身经验有选择的进行, 避免肝静脉撕裂引 起出血和气体栓塞的风险. 如果切肝过程中出现肝静 脉出血可以通过压迫、降低中心静脉压等方法控制出 血速度后予以热烧灼或缝扎止血.

通过有效的肝脏血流预控，可以实现拟切除肝脏 区域内的无血状态，同时保持其余肝脏正常的血流供 应, 切肝的过程变得更加的从容, 不用为了缩短肝门阻 断时间而加快肝脏离断速度, 从而使得切肝过程中的 精细解剖成为可能, 有效减少了术中出血, 降低术后并

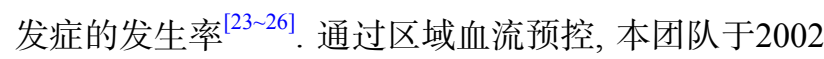
年报道国内首例腹腔镜左半肝切除 ${ }^{[27]}, 2005$ 年报道国 内首例腹腔镜肝右三叶切除 ${ }^{[28]}, 2010$ 年首次提出模式 化腹腔镜肝左外叶切除 ${ }^{[29]}$. 与传统的开腹手术相比, 腹 腔镜肝切除术中平均出血量、术后并发症及平均住院 日均明显减少 ${ }^{[30,31]}$.

\section{2 入路选择}

外科手术首先就会面临入路的问题，手术入路不 单纯是指实施操作的路径选择, 而是包含了通道、路 径、目标显露在内的一系列策略规划. 正确的手术人 路不仅能够使手术事半功倍, 减轻医源性损伤和并发 症, 还能够简化手术操作, 便于手术的实施与推广. 其 中比较有代表性的是胰腺手术的人路选择, 因为胰腺 的解剖位置深在, 周围毗邻的脏器及血管结构复杂, 不 仅手术难度大，而且术后严重并发症发生率也高于其 他手术.

本团队针对胰腺病灶的不同部位和微创手术的特 点, 建立了个体化的微创胰腺手术人路体系, 包括: (1) 经后腹膜人路的胰腺手术 ${ }^{[2 \sim 37]}$ (图3). 首先, 将后腹腔镜 技术引人到胰腺手术领域, 利用后腹膜人路不干扰正 常腹腔解剖结构的特点, 更直接地到达操作区域, 减少 了手术操作和路径创伤. 目前已开展的手术方式有胰 岛素瘤剜除术、胰体尾切除术、胰腺坏死组织清除引 流术等 ${ }^{[32,34,36,38]}$, 其中胰岛素瘤剜除及胰体尾切除术的 手术时间30 100 min，术中出血10 100 mL，术后仅个 
(a)

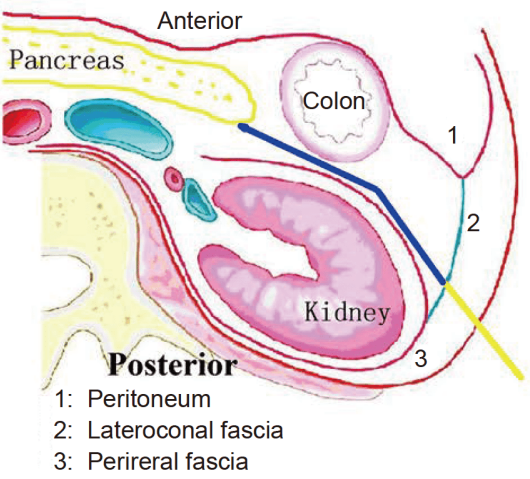

(b)

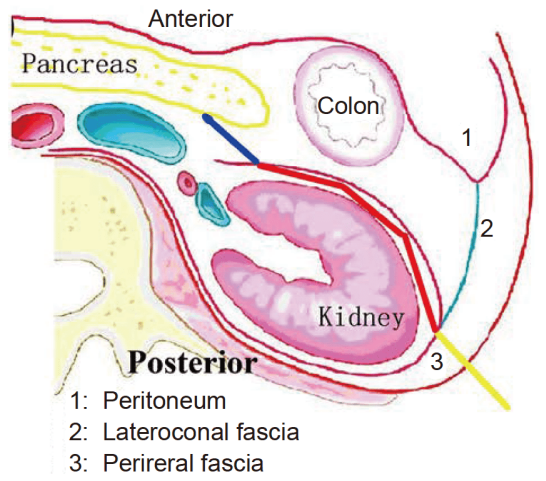

图 3 (网络版彩色)经后腹膜人路的胰腺手术 ${ }^{[34]}$. (a) 旁侧人路(经过肾旁后间隙、直接人肾旁前间隙); (b) 后侧人路(经过肾旁后间隙、肾周间 隙, 最后人肾旁前间隙)

Figure 3 (Color online) Two pathways for retroperitoneoscopic pancreatic suregy ${ }^{[34]}$. (a) Lateral approach (posterior pararenal space to anterior pararenal space); (b) posterior approach (posterior pararenal space, perirenal space to anterior pararenal space)

别患者并发A级胰瘘, 无其他严重并发症, 平均术后7 d 即可出院 ${ }^{[33]}$, 较常规入路优势明显. 对于重症胰腺炎患 者，后腹腔镜手术具有明显的损伤控制优势. (2) 经小 网膜囊人路的胰腺手术 ${ }^{[39]}$. 当肿瘤位于胰腺颈体部上 方时, 常规的手术人路需切开胃结肠韧带. 由于该人路 的路径长、切开组织较多, 当大网膜肥厚或黏连时, 极 易出现网膜血管出血甚至损伤结肠而导致中转开腹. 利用小网膜人路可以快速、安全地显露胰腺颈体部肿 瘤, 不仅简化了操作, 还能避免过多的组织和血管损伤, 术后恢复迅速. (3) 经脾结肠韧带入路的胰腺手术 ${ }^{[40]}$. 对于胰尾部的肿瘤，传统手术人路也存在游离范围 大、涉及组织较多的问题，容易影响患者的术后康复. 利用能量器械直接离断脾结肠韧带, 能迅速、安全地 显露胰尾病灶，有效降低了微创胰尾部肿瘤的手术难 度. (4) 经L， R孔入路的微创胰十二指肠切除术 ${ }^{[14,41]}$. 开腹胰十二指肠切除的胃肠吻合术通常行结肠前吻合,

(a)

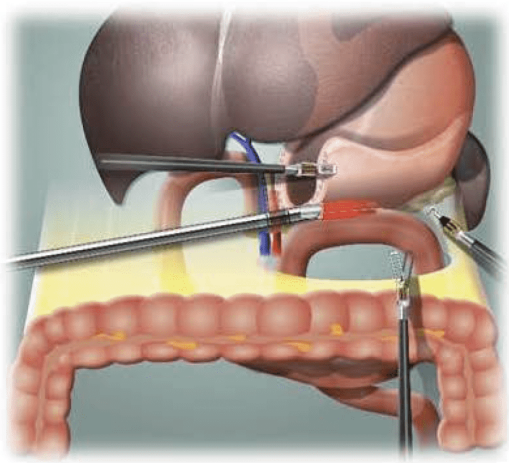

但是在微创条件下翻转横结肠操作存在困难, 当患者 横结肠粗大或网膜脂肪较多时吻合难度明显增加. 胰 腺钩突的处理一直是胰十二指肠切除术中的难点，充 分的钩突显露是减少出血、保证切缘的前提. 基于微 创手术空间有限、操作活动度小以及仰视视角的特点, 本团队探索出了简化操作、提高效率的最优路径: L孔 和R孔人路(图4). L孔入路：从横结肠下方、结肠中血 管左侧打开横结肠系膜，建立 $\mathrm{L}$ ， 经 $\mathrm{L}$ 孔可将近端空 肠提至胃后壁行胃肠吻合术. 该人路无需翻转横结肠, 是行胃肠吻合的最短路径，吻合口的张力小，简便易 行，目前已成为本团队微创胃肠吻合的常规人路. $R$ 孔 人路：从横结肠下方、结肠中血管右侧打开横结肠系 膜，建立 $\mathrm{R}$ 孔，经该孔可直接暴露胰腺钩突和十二指肠 降段和水平段, 便于钩突肿瘤的局部剜除, 降低了钩突 血管处理和黏连组织游离的难度. 个体化微创胰腺手 术人路体系的建立，充分利用了微创手术的优势，避免

(b)

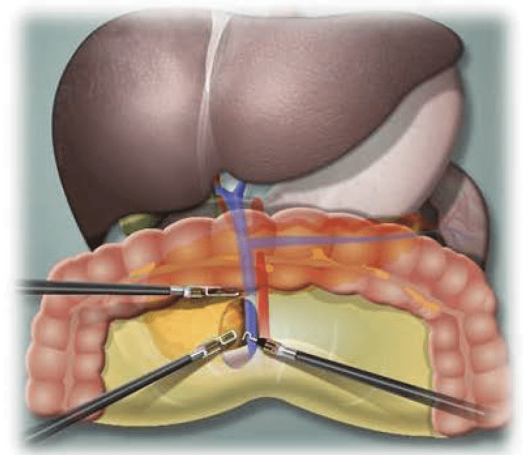

图 4 (网络版彩色) 经L、R孔人路的胰腺手术. (a) L孔人路; (b) R 孔人路

Figure 4 (Color online) Pancreatic surgery through L and R hole approach. (a) L-hole approach; (b) R-hole approach 
了过多的医源性损伤，根据肿瘤的不同位置“因地制 宜”, 简化了手术操作, 降低了并发症的风险, 有利于患 者的快速康复和微创胰腺手术的应用与推广.

\section{3 切除技术}

切除是手术过程中的重要环节, 切除的彻底性和 可操作性是需要外科医生关注的关键问题, 对于微创 手术来说更是如此. 以胰腺癌根治为例, 为了防止胰腺 癌术后复发和转移、改善患者预后, 有学者提出了胰 腺手术中的整块切除理念, 即包括胰腺及胰周淋巴 结、脂肪组织层在内的整块切除 ${ }^{[42]}$, 通过术中的整块 切除达到提高R0切除率. 本团队提倡将整块切除的理 念应用在微创胰腺癌根治中. (1) 在胰十二指肠切除 时, 我们常规将 $8 a, 8 p, 12 a, 12 b$ 和 $12 p$ 组淋巴结一并清 扫. 术中需要以肝总动脉/肝固有动脉和门静脉为引导, 将左侧肝十二指肠韧带附近淋巴结充分游离后, 与 $12 p$ 淋巴结一并汼拉至门静脉右后方待整块切除. 在离断 钩突时, 自足侧向头侧紧贴肠系膜上动脉、腹主动脉 和腹腔干右侧壁离断钩突系膜和神经淋巴组织, 最后 将标本连同淋巴结en-block整块切除 ${ }^{[14]}$. 该“两掏一翻” 的淋巴结整块切除法, 充分利用腹腔镜和机器人镜头 的放大优势和侧方视角，在保证R0切除的同时，实现 淋巴结的整块和完整切除是安全、可行的 ${ }^{[43]}$. (2) 针对 传统胰体尾切除手术对胰腺后背膜与肾筋膜间组织切 除不彻底, 术后肿瘤易复发的情况, 根治性顺行模块化 胰脾切除(radical antegrade modular pancreatosplenectomy，RAMPS)手术更强调包括肾筋膜、肾前脂肪囊甚 至左侧肾上腺等深部组织的切除和清扫, 能够提高 R0 切除率并改善患者预后 ${ }^{[44]}$. 本团队在国内较早将 RAMPS手术应用在腹腔镜和机器人微创外科领域，利 用微创手术中高清镜头的放大效果和微创器械的精细 操作, 实现更高的R0切除率、更多的淋巴结切除和更 彻底的清扫范围, 从而有效降低术后复发率, 延长患者 生存时间. 本团队报道行腹腔镜和机器人RAMPS术后, R0切除率达到 $97.1 \%$ 和 $100 \%$ ，淋巴结清扫数目分别为 10.5 和 11 枚, 术后总生存时间可达 25 和 27 个月 ${ }^{[44-46]}$.

随着腔镜设备和技术的发展, 微创外科的适应症 得以不断拓宽. 目前腹腔镜手术已经能够覆盖几乎所 有的肝脏外科术式, 但由于各个医院操作习惯及手术 风格的差异, 从Trocar布局、手术人路到手术器械的选 择、肝脏的离断方法均有显著差异, 不仅不利于手术 的实施和推广, 也不利于手术质量的统一和疗效的评
价. 本团队根据不同术式的特点, 分别制定了左外叶、 左半肝、右半肝切除等模式化手术步骤 ${ }^{[4749]}$, 力求实 现开腹手术微创化和复杂手术简单化. 通过对手术步 骤的简化和归纳, 更进一步有利于模式化切肝技术在 广大基层医院的推广和应用, 有利于微创肝切除水平 的共同提高. 将肝切除术分解为 7 个步骤进行: (1) 患者 取平卧位，常规4孔法操作，具体体位和操作孔布局可 根据手术需要调整; (2) 按序离断肝周韧带, 游离相应 肝叶; (3) 粗分离进人目的肝段或肝叶血管蒂, 并结扎 或切割离断; (4) 根据选取切割线离断肝组织; (5) 粗分 离出相应肝静脉, 切割离断; (6) 处理肝断面; (7) 留置引 流，取出标本. 通过定量化描述肝内管道的空间分布， 将管道连同实质组织进行“集束化”离断，将腹腔镜肝 左外叶切除简化成“三步断肝法”, ${ }^{[49]}$ (图5), 避免了肝门 解剖的高风险, 降低了手术难度, 让腹腔镜肝左外叶切 除普及到县级医院. 对于相对难度较大、对腔镜技术 要求较高的腹腔镜右半肝切除术, 围手术期数据显示: 平均手术时间(256.17 \pm 71.16$) \mathrm{min}$, 平均出血量(493.48土 $239.94) \mathrm{mL}$, 术后平均住院时间(8.91 \pm 3.6$) \mathrm{d}, 1$ 例术后出 现胆瘘, 1 例出现切口感染, 未发生严重并发症, 手术时 间与开腹手术比较而言无明显增加，术后住院时间则 明显缩短了, 有利于患者的术后恢复 ${ }^{[48]}$. 模式化的肝切 除术化繁为简, 更加安全、简便, 重复性好, 更便于腹 腔镜肝切除术的推广和广大同道的学习.

\section{4 重建技术}

切除手术后的重建质量直接影响了术后并发症的 发生率, 与患者预后息息相关. 特别是胰十二指肠切除 术，由于其需要完成胰肠(胰胃)、胆肠、胃肠三个吻 合, 已成为腹部外科难度最大、风险最高的术式. 胰腺 吻合重建是其中最具挑战的消化道重建方式，是目前
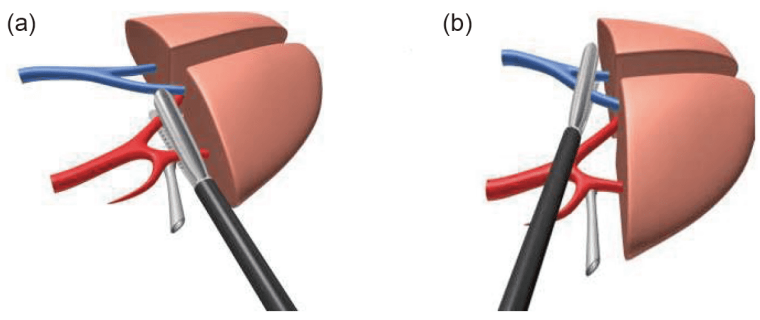

图 5 (网络版彩色) 模式化肝左外叶切除术 ${ }^{[49]}$. (a) 离断人肝血流; (b) 离断出肝血流

Figure 5 (Color online) A modeling method for laparoscopic left lateral hepatectomy ${ }^{[49]}$. (a) Hepatic inflow dissection; (b) hepatic outflow dissection 

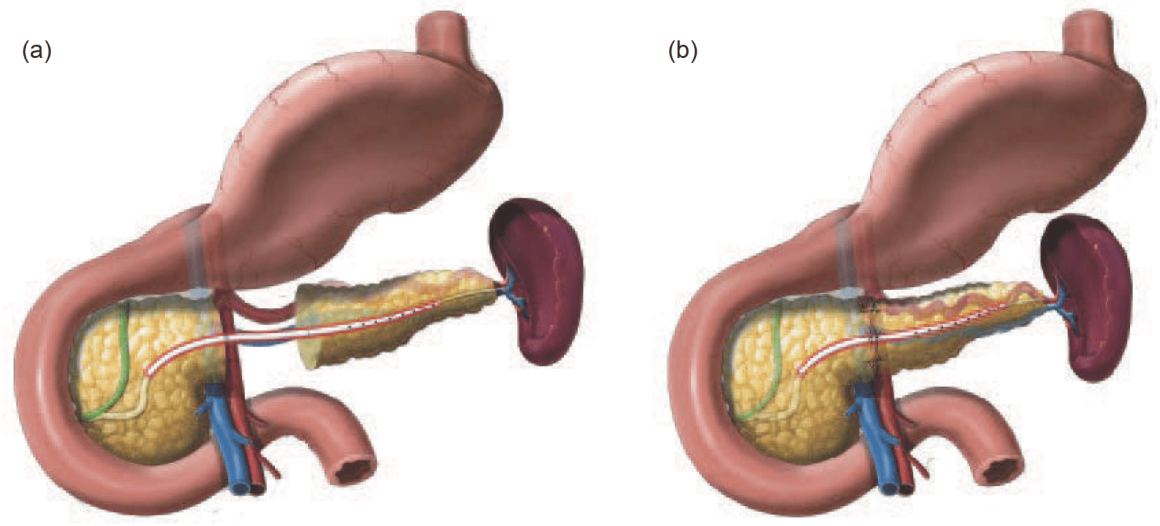

图 6 (网络版彩色)胰腺端端吻合重建术 ${ }^{[19]}$. (a) 主胰管架桥修复; (b) 胰腺端端吻合

Figure 6 (Color online) Pancreatic end-to-end anastomotic reconstruction ${ }^{[19]}$. (a) Bridging repair of the main pancreatic duct; (b) pancreatic end-toend anastomotic

阻碍微创胰十二指肠手术发展的主要原因 ${ }^{[50,51]}$. 尽管 外科医生在胰腺吻合重建方式和手术技术方面做出了 多种的尝试, 各种新型吻合层出不穷, 然而到目前为止 尚没有一种重建方式能够完全避免胰瘘 ${ }^{[52]}$. 本团队结 合在开腹和微创胰腺手术中的大量经验, 改变了追求 严密机械性缝合的传统理念，从保护吻合口血供、降 低吻合口张力、减少对胰腺穿透性伤害的角度出发, 对吻合方法不断优化，提出了简便易行的单针全层胰 肠吻合法，即301式胰肠吻合 ${ }^{[53]}$. 其核心技术采用U型 缝合胰腺残端，减少胰腺断面上分支胰管的外渗; 1 针 4-0 prolene线连续缝合胰腺残端全层和空肠浆肌层; 用 主胰管与空肠黏膜的对合方法代替黏膜对黏膜吻合. 此方法简单易行, 术后胰瘘发生率低, 具有良好的安全 性和有效性，本团队应用 $1+2$ 胰肠吻合的机器人胰十二 指肠切除术后临床相关胰瘘发生率为 $9.35 \%{ }^{[41,54]}$, 而应
用301式胰肠吻合术后仅出现生化漏, 未见B级和C级胰 瘘 ${ }^{[53]}$, 显著低于文献报道开腹 $(16.3 \%)$ 、腹腔镜 $(12.1 \%)$ 和机器人 $(11 \%)$ 手术的胰漏发生率 ${ }^{[5]}$.

传统的手术理念中，当胰腺肿瘤剜除术中胰管损 伤时, 需要行胰肠吻合术、挽救性远端胰腺切除术、 甚至是胰十二指肠切除术. 为了减少手术创伤、尽可 能保护胰腺功能, 本团队率先尝试在机器手术系统下 实施主胰管架桥修复，避免了挽救性胰腺切除手术带 来的巨大创伤 ${ }^{[17,56]}$. 由此术式启发, 参考工程建筑中的 桥梁建造过程, 本团队创立了创新性桥梁合拢理论, 针 对胰腺中段切除可行胰腺端端吻合重建术(图6)，术后 患者恢复良好, 无严重术后并发症. 该技术能够最大程 度地保留正常胰腺组织, 保证胰腺生理和解剖的完整 性, 有利于患者的远期预后, 有望改变胰腺良性疾病的 传统外科治疗方法 ${ }^{[19]}$.

\section{参考文献}

1 Yan X Y, Dong C Y, Yao C. The evolvement of evidence-based medicine research in the big data era (in Chinese). Chin J Evid-Based Med, 2017, 17: 249-254 [阎小妍, 董冲亚, 姚晨. 大数据时代的循证医学研究. 中国循证医学杂志, 2017, 17: 249-254]

2 Liu R. Medical intervention should focus on prognosis: Dynamic prognosis-control in medicine (in Chinese). Acad J Chin PLA Med Sch, 2018, 39: 931-933 [刘荣. 医疗干预应聚焦预后一一医学中的动态预后控制. 解放军医学院学报, 2018, 39: 931-933]

3 Liu R. The transformation of doctors in the era of intelligent medicine (in Chinese). J Laparo Surg, 2018, 23: 1-3 [刘荣. 智能医学时代医生的转 型. 腹腔镜外科杂志, 2018, 23: 1-3]

4 Liu R. Enter the new era of intelligent medicine (in Chinese). Chin J Laparo Surg (Electr Ed), 2018, 11: 65-67 [刘荣. 走进智能医学新时代. 中华 腔镜外科杂志(电子版), 2018, 11: 65-67]

5 Liu R. Opportunities and challenges of intelligent surgery (in Chinese). Chin J Laparo Surg (Electr Ed), 2017, 10: 327-329 [刘荣. 智能外科的机 遇与挑战. 中华腔镜外科杂志(电子版), 2017, 10: 327-329]

6 Liu H. On the prognosis (in Chinese). Med Philos, 2002, 4-8 [刘虹. 论预后. 医学与哲学, 2002, 4-8]

7 Liu R, Liu Q. Intraoperative risk precontrol and minimally invasive hepato-pancreato-biliary surgery (in Chinese). Chin J Laparo Surg (Electr Ed), 2017, 10: 65-67 [刘荣, 刘渠. 术中风险预控与肝胆胰微创外科. 中华腔镜外科杂志(电子版), 2017, 10: 65-67] 
8 Chen W, Ji H, Feng J, et al. Classification of pancreatic cystic neoplasms based on multimodality images. Mach learn med imaging, 2018, 11046: 161-169

9 Ackerman M J. The visible human project: A resource for education. Acad Med, 1999, 74: 667-670

10 Mezger U, Jendrewski C, Bartels M. Navigation in surgery. Langenbecks Arch Surg. 2013, 398: 501-514

11 Zhang W Q, Dai K R, Wang C T. The present state and development of navigation system for orthopedic surgery (in Chinese). J Med Biomech, 2004, 19: 51-55 [张文强, 戴攰戎, 王成奉. 外科手术导航系统的研究现状及进展. 医用生物力学, 2004, 19: 51-55]

12 Schicho K, Figl M, Donat M, et al. Stability of miniature electromagnetic tracking systems. Phys Med Biol, 2005, 50: 2089-2098

13 Qi S T, Liu T B. Surgical robot system and its clinical applications (in Chinese). Chin Med Dev, 2011, 26: 56-59 [戚仕涛, 刘铁兵. 外科手术机器 人系统及其临床应用. 中国医疗设备, 2011, 26: 56-59]

14 Liu R. Establishment and technical optimization of LR robotic pancreaticoduodenectomy (in Chinese). Chin J Laparo Surg (Electr Ed), 2016, 9: 193-195 [刘荣. LR式机器人胰十二指肠切除术手术方法建立和技术优化. 中华腔镜外科杂志(电子版), 2016, 9: 193-195 ]

15 Liu R. Tunnel construction strategy in minimally invasive pancreatic surgery (in Chinese). Chin J Laparo Surg (Electr Ed), 2018, 11: 193-195 [刘 荣. 胰腺手术中的“隧道构建”策略. 中华腔镜外科杂志(电子版), 2018, 11: 193-195]

16 Liu R, Zhao G D, Ma X, et al. The first case of robotic retroperitoneal laparoscopic pancreatic surgery (in Chinese). Chin J Laparo Surg (Electr Ed), 2016, 9: 59-60 [刘荣, 赵国栋, 马釒金, 等. 机器人后腹腔镜胰腺手术首例报道. 中华腔镜外科杂志(电子版), 2016, 9: 59-60]

17 Liu R, Zhao G D, Yin Z Z, et al. A case report of robotic pancreatic tumor enucleation combined with main pancreatic duct bridge repair (in Chinese). Chin J Laparo Surg (Electr Ed), 2016, 9: 373-374 [刘荣, 赵国栋, 尹注增, 等. 机器人胰腺肿瘤剜除联合主胰管架桥修复术个案报道. 中华腔镜外科杂志(电子版), 2016, 9: 373-374]

18 Liu R, Wang Z Z, Gao Y X, et al. Rong's robotic middle pancreatectomy: A case report (in Chinese). Chin J Laparo Surg (Electr Ed), 2017, 5: 319320 [刘荣, 王子政, 高元兴, 等. 机器人“荣氏”胰腺中段切除术一例报道. 中华腔镜外科杂志(电子版), 2017, 5: 319-320]

19 Liu R, Zhao G D. Promotion of innovative theory of bridge closure in changing traditional surgical approaches for benign pancreatic diseases (in Chinese). Chin J Gen Surg, 2018, 27: 263-268 [刘荣, 赵国栋. 创新性桥梁合拢理论对改变胰腺良性病外科手术方法的推动. 中国普通外科杂 志, 2018, 27: 263-268]

20 Liu R. The rational selection and evaluation of surgical pathway of laparoscopic liver resection (in Chinese). Chin J Laparo Surg (Electr Ed), 2018, 11: 129-131 [刘荣. 腹腔镜肝切除手术人路合理选择及评价. 中华腔镜外科杂志(电子版), 2018, 11: 129-131]

21 Liu R, Zhao G D. New concepts of the anatomy of the liver: From the trunk theory of cadaveric anatomy to the basin theory of clinical potential morphology (in Chinese). Chin J Laparo Surg (Electr Ed), 2018, 11: 257-260 [刘荣, 赵国栋. 肝脏解剖: 从尸体静态解剖学下的树干理论到临床 潜能形态学下的流域学说. 中华腔镜外科杂志(电子版), 2018, 11: 257-260]

22 Liu R, Hu M G. Main points of technique for laparoscopic right hemihepatectomy (in Chinese). Chin J Pract Surg, 2010, 30: 654-656 [刘荣, 胡明 根. 腹腔镜下右半肝切除的技术要点. 中国实用外科杂志, 2010, 30: 654-656]

23 Liu R. Laparoscopic regular hepatectomy (in Chinese). J Surg Concepts Pract, 2004, 9: 450-452 [刘荣. 腹腔镜下规则性肝切除. 外科理论与实 践, 2004, 9: 450-452]

24 Liu R. The study on development and current status of totally laparoscopic hepatectomy in China mainland: 14 years' experience in multiple institutions (in Chinese). Chin J Laparo Surg (Electr Ed), 2009, 2: 5-13 [刘荣. 中国大陆地区完全腹腔镜肝脏切除术发展及现状: 多中心14年 经验. 中华腔镜外科杂志(电子版), 2009, 2: 5-13]

25 Liu R, Wang Y H, Zhou N X, et al. Laparoscopic anatomical resection of left liver: Report of 3 cases (in Chinese). Chin J Pract Surg, 2003, 23: 556-557 [刘荣, 王悦华, 周宁新, 等. 完全腹腔镜解剖性左半肝切除3例报告. 中国实用外科杂志, 2003, 23: 556-557]

26 Wang Y H, Liu R, Zhou N X, et al. Anatomical basis and techniques of laparoscopic anatomical hepatectomy (in Chinese). Chin J Gen Surg, 2003, 7: 17-19 [王悦华, 刘荣, 周宁新, 等. 完全腹腔镜规则性肝切除的解剖基础与技术问题. 中华普通外科杂志, 2003, 7: 17-19]

27 Liu R, Wang Y H, Zhou N X, et al. Laparoscopic left hepatectomy: A case report (in Chinese). Chin J Pract Surg, 2002, 10: 61 [刘荣, 王悦华, 周宁 新, 等. 腹腔镜左半肝切除术 1 例报告. 中国实用外科杂志, 2002, 10: 61]

28 Liu R, Hu M G, Wang G. One case of complete laparoscopic right Hepatic Trisegmentectomy (in Chinese). Natl Med J Chin, 2005, 25: 1783 [刘荣, 胡明根, 王刚. 完全腹腔镜肝右三叶切除一例. 中华医学杂志, 2005, 25: 1783]

29 Liu R, Zhao G D. The "gold standard” method of left hepatic lobectomy: Laparoscopic left hepatic lobectomy (in Chinese). Chin J Laparo Surg (Electr Ed), 2010, 3: 474-478 [刘荣, 赵国栋. 肝左外叶切除“金标准”术式: 腹腔镜肝左外叶切除术. 中华腔镜外科杂志(电子版), 2010, 3: 474-478]

30 Wang G, Liu R. Clinical study of laparoscopic versus open hepatectomy (in Chinese). Chin J Pract Surg, 2005, 10: 617-620 [王刚, 刘荣. 腹腔镜和 开腹肝切除的临床对比研究. 中国实用外科杂志, 2005, 10: 617-620]

31 Wang X, Hu M, Zhao Z, et al. An improved surgical technique for pure laparoscopic left hemihepatectomy: Ten years experience in a tertiary center. J Laparoendosc Adv Surg Tech A, 2016, 26: 862-869

32 Liu R, Zhang X, Zhao G D, et al. Retroperitoneal laparoscopic pancreatic surgery: From hypothesis to clinical application (in Chinese). Chin J Laparo Surg (Electr Ed), 2011, 4: 426-430 [刘荣, 张旭, 赵国栋, 等. 后腹腔镜胰腺手术: 从假想到临床应用. 中华腔镜外科杂志(电子版), 2011, 
4: 426-430]

33 Zhao G D, Liu R, Ma X, et al. Retroperitoneoscopic pancreatectomy: A report of 4 cases (in Chinese). Chin J Pract Surg, 2012, 32: 85-87 [赵国栋, 刘荣, 马崟, 等. 后腹腔镜胰腺切除术4例报告. 中国实用外科杂志, 2012, 32: 85-87]

34 Zhao G, Hu M, Liu R, et al. Two anatomical pathways for retroperitoneoscopic pancreatectomy: Indications for the posterior and lateral approaches. World J Surg, 2014, 38: 3023-3032

35 Zhao G, Hu M, Liu R, et al. Single-port retroperitoneoscopic pancreatectomy: Preliminary results from the first 3 patients. J Clin Gastroenterol, 2014, 48: 559-562

36 Zhao G, Xue R, Ma X, et al. Retroperitoneoscopic pancreatectomy: A new surgical option for pancreatic disease. Surg Endosc, 2012, 26: 16091616

37 Zhao G, Wang Z, Hu M, et al. Preliminary clinical experience with robotic retroperitoneoscopic pancreatic surgery. World J Surg Oncol, 2018, 16: 171

38 Zhao G, Hu M, Liu R, et al. Retroperitoneoscopic anatomical necrosectomy: A modified single-stage video-assisted retroperitoneal approach for treatment of infected necrotizing pancreatitis. Surg Innov, 2015, 22: 360-365

39 Liu R, Xu D B, Luo Y, et al. One case of laparoscopic resection of small omentum cyst (in Chinese). Natl Med J Chin, 2007, 48 : 3456 [刘荣, 许大 彬, 罗英, 等. 腹腔镜小网膜囊肿切除一例. 中华医学杂志, 2007, 48: 3456]

40 Liu R, Yin Z Z, Zhao G D, et al. Splenocolic ligament approach for laparoscopic or robotic distal pancreatectomy (in Chinese). J Laparo Surg, 2017, 22: 66-69 [刘荣, 尹注增, 赵国栋, 等. 脾结肠韧带人路的腹腔镜或机器人胰尾肿瘤切除术. 腹腔镜外科杂志, 2017, 22: 66-69]

41 Liu R, Zhao G D, Yin Z Z. Technique and theory of LR's $1+2$ pancreaticojejunostomy in robotic pancreatectomy: An analysis of 104 cases (in Chinese). Chin J Laparo Surg (Electr Ed), 2017, 10: 7-10 [刘荣, 赵国栋, 尹注增. 机器人LR式1+2胰肠吻合方法的理论与技巧: 附104例病例报 道. 中华腔镜外科杂志(电子版), 2017, 10: 7-10]

$42 \mathrm{Xu} \mathrm{D}$, Jiang K R, Lu Z P, et al. Clinical effect of radical antegrade modular pancreatosplenectomy for carcinoma of pancreatic body and tail (in Chinese). Chin J Dig Surg, 2016, 15: 567-573 [徐冬, 蒋奎荣, 陆子鹏, 等. 根治性顺行模块化胰脾切除术治疗胰体尾癌的临床疗效. 中华消化 外科杂志, 2016, 15: 567-573]

43 Liu R, Zhang T, Zhao Z M, et al. The surgical outcomes of robot-assisted laparoscopic pancreaticoduodenectomy versus laparoscopic pancreaticoduodenectomy for periampullary neoplasms: A comparative study of a single center. Surg Endosc, 2017, 31: 2380-2386

44 Qu L, Zhiming Z, Xianglong T, et al. Short- and mid-term outcomes of robotic versus laparoscopic distal pancreatosplenectomy for pancreatic ductal adenocarcinoma: A retrospective propensity score-matched study. Int J Surg, 2018, 55: 81-86

45 Liu R, Liu Q, Zhao Z M, et al. Robotic versus laparoscopic distal pancreatectomy: A propensity score-matched study. J Surg Oncol, 2017, 116: $461-469$

46 Zhang T, Zhao Z M, Gao Y X, et al. The learning curve for a surgeon in robot-assisted laparoscopic pancreaticoduodenectomy: A retrospective study in a high-volume pancreatic center. Surg Endosc, 2018, doi: 10.1007/s00464-018-6595-0

47 Wang X F, Hu M G, Zhao G D, et al. Stylized laparoscopic left hemihepatectomy: Consideration as a standard laparoscopic procedure (in Chinese). Chin J Laparo Surg (Electr Ed), 2014, 7: 160-166 [王雪飞, 胡明根, 赵国栋, 等. 一种腹腔镜标准术式的探索: 模式化腹腔镜左半肝切除术. 中 华腔镜外科杂志(电子版), 2014, 7: 160-166]

48 Xu Y, Hu M G, Zhao G D, et al. Stylized laparoscopic right hemihepatectomy (in Chinese). Chin J Laparo Surg (Electr Ed), 2015, 8: 237-241 [许 勇, 胡明根, 赵国栋, 等. 模式化腹腔镜右半肝切除术. 中华腔镜外科杂志(电子版), 2015, 8: 237-241]

49 Zhao G D, Hu M G, Liu R. A modeling method for laparoscopic left lateral segment liver resection: Report of 71 cases (in Chinese). J South Med Univ, 2011, 31: 737-740 [赵国栋, 胡明根, 刘荣. 模式化腹腔镜肝左外叶切除术: 附71例临床应用报道. 南方医科大学学报, 2011, 31: 737-740]

50 Zureikat A H, Postlewait L M, Liu Y, et al. A multi-institutional comparison of perioperative outcomes of robotic and open pancreaticoduodenectomy. Annal Surg, 2016, 264: 640-649

51 Chen Y J, Lai E C, Lau W Y, et al. Enteric reconstruction of pancreatic stump following pancreaticoduodenectomy: A review of the literature. Int J Surg, 2014, 12: 706-711

52 Pedrazzoli S. Pancreatoduodenectomy (PD) and postoperative pancreatic fistula (popf): A systematic review and analysis of the popf-related mortality rate in 60,739 patients retrieved from the english literature published between 1990 and 2015. Medicine (Baltimore). 2017, 96: e6858

53 Liu R, Liu Q, Zhao Z Z, et al. Application of a novel technique of pancreaticojejunostomy in pancreaticoduodenectomy (in Chinese). J Laparo Surg, 2018, 23: 854-857 [刘荣, 刘渠, 赵之明, 等. 单针全层胰肠吻合(301式)在胰十二指肠切除术中的应用. 腹腔镜外科杂志, 2018, 23: 854-857]

54 Liu R, Zhao G D, Tang W B, et al. A single-team experience with robotic pancreatic surgery in 1010 cases (in Chinese). J South Med Univ, 2018, 38: 130-134 [刘荣, 赵国栋, 唐文博, 等. 机器人胰腺手术1010例经验与教训. 南方医科大学学报, 2018, 38: 130-134]

55 Shin S H, Kim Y J, Song K B, et al. Totally laparoscopic or robot-assisted pancreaticoduodenectomy versus open surgery for periampullary neoplasms: Separate systematic reviews and meta-analyses. Surg Endosc, 2017, 31: 3459-3474

56 Liu R, Wang Z Z, Gao Y X, et al. Application of end-to-end anastomosis in robotic central pancreatectomy. J Vis Exp, 2018, doi: 10.3791/57495 
Summary for “预后控制外科: 从理论到实践”

\title{
Prognosis control surgery: From theory to practice
}

\author{
Rong Liu*, Qu Liu, Fei Wang, Zizheng Wang, Tao Zhang \& Gong Zhang \\ Military Institute of Hepatobiliary Surgery, Second Department of Hepatopancreatobiliary Surgery, Chinese People's Liberation Army (PLA) General \\ Hospital, Beijing 100853, China \\ * Corresponding author: E-mail: liurong301@126.com
}

Rapid advances of big data and artificial intelligence (AI) techniques have pushed the envelope of traditional surgery to intelligent surgery, which revolutionizes the traditional medicine model dominated by the experiences of interveners and evidence-based medicine. Intelligent surgery requires to judiciously integrate the clinical experience of interveners and medical evidences with the new AI techniques, so as to achieve the best therapeutic effect for patients through effective disease risk control. This paper puts forward a new theory of prognostic control surgery in the long-term clinical practice of hepato-pancreato-biliary surgery. Our theory takes the optimal prognosis of patients as the goal, and pre-controls the disease risk through the best combination of the optimal intervener, intervention methods and intervention timing to achieve the maximum clinical benefits of patients with minimal medical trauma. This theory also takes full advantage of information technologies such as neural networks, deep learning, big data and imaging system, and uses high-dimensional network decision making to replace the traditional two-dimensional decision making tree model.

A main technical challenge in the theory is how to effectively exploit the AI techniques in to improve the production efficiency of large hospitals, enhance the service level of primary medical care, and improve the diagnosis, prediction, treatment of diseases. Prognostic control surgery advocates the exploitation of emerging information technology methods, such as medical image recognition and prediction, 3D reconstruction based surgical planning, intraoperative navigation, and remote intelligent robotic surgical system, to promote the rapid and balanced surgical diagnosis and treatment.

Guided by the theory of prognosis control surgery, our team successfully established minimally invasive anatomic hepatectomy, individualized pancreatic surgical approaches, modular hepatectomy, single-layer continuous suture of pancreaticojejunostomy, and end-to-end pancreatic anastomosis and advocated en-bloc resection of pancreatic cancer. This paper also provides the details of four core surgical strategies proposed in our prognosis control surgery, namely, blood control technique, surgical approach selection, resection technique and reconstruction technique, by which to achieve the minimally invasive surgery regularization and the complex surgery simplification.

prognosis control surgery, blood control technique, surgical approach selection, resection technique, reconstruction technique, artificial intelligence

doi: 10.1360/N972019-00141 